\title{
Atresia de esôfago: diagnóstico perinatal e assistência perioperatória - protocolo da Maternidade Escola Assis Chateaubriand
}

\section{Esophageal atresia: perinatal diagnosis and perioperative care - a Maternity School Assis Chateaubriand protocol}

Geysa Maria Monte Saraiva Câmara ${ }^{1}$. Liliana Soares Nogueira Paes².Eveline Campos Monteiro Castro ${ }^{3}$. Thyago Araújo Fernandes ${ }^{4}$. Antônio Aldo Melo Filho ${ }^{5}$. Osvaldo Barros Rebelo Neto ${ }^{6}$.

1 Médica Pediatra, Residente de Neonatologia, Maternidade Escola Assis Chateaubriand (MEAC), Fortaleza, Ceará, Brasil. 2 Neonatologista, Supervisora do Programa de Residência Médica em Neonatologia, Maternidade Escola Assis Chateaubriand (MEAC), Fortaleza, Ceará, Brasil. 3 Doutora em Pediatria, Universidade Federal de São Paulo (UNIFESP), Neonatologista e Chefe da Unidade Neonatal, Maternidade Escola Assis Chateaubriand (MEAC), Fortaleza, Ceará, Brasil. 4 Anestesiologista, Hospital Universitário Walter Cantídio (HUWC), Universidade Federal do Ceará (UFC), Fortaleza, Ceará, Brasil. 5 Doutor em Cirurgia, Universidade Estadual de Campinas (UNICAMP), Professor Adjunto do Departamento de Cirurgia, Universidade Federal do Ceará (UFC), Fortaleza, Ceará, Brasil. 6 Cirurgião Pediátrico, Maternidade Escola Assis Chateaubriand (MEAC). Chefe da Cirurgia Pediátrica, Hospital Universitário Walter Cantídio (HUWC), Universidade Federal do Ceará (UFC), Fortaleza, Ceará, Brasil.

\section{RESUMO}

Atribui-se a Gibson, na segunda metade do século XVII, a clássica descrição da atresia de esôfago (AE), malformação congênita de incidência de um caso a cada 2500-4500 nascimentos. Sua etiologia permanece desconhecida, porém, estudos associam-na com prematuridade, gemelaridade e consanguinidade parental. Mediante localização do sítio de atresia e identificação de fístula traqueal associada, pode ser classificada em cinco variantes, cuja compreensão determina o manejo clínico-cirúrgico. Trata-se de uma condição benigna, quando dissociada de outras anomalias congênitas graves, com sobrevida aproximada de 95\% em alguns centros. Descreve-se a seguir o protocolo de condutas da Maternidade Escola Assis Chateaubriand, empregado no diagnóstico e nas rotinas cirúrgicas perioperatórias diante de casos de atresia de esôfago.

Palavras-chave: Atresia esofágica. Perinatologia. Diagnóstico. Assistência perioperatória. Protocolos.

\section{ABSTRACT}

In the second half of the seventeenth century, Gibson is credited with the first description of esophageal atresia (EA), a congenital malformation that occurs in about 1 per 2500-4500 births. The etiology of EA remains unknown, but several studies point out prematurity, gemelarity and parental consanguinity as major risk factors. Once located the site of atresia and identification of associated tracheal fistula, it can be classified into five variants, an important step towards understanding the clinical-surgical management. It is a benign condition when other severe congenital anomalies are not present, its survival rate reaches $95 \%$ in some centers. We describe the protocol of routines of the Maternidade Escola Assis Chateaubriand, used for the diagnosis and the perioperative surgical management in cases of esophageal atresia.

Keywords: Esophageal atresia. Perinatology. Diagnosis. Perioperative care. Protocols.

Autor correspondente: Geysa Maria Monte Saraiva Câmara, Rua Barbosa de Freitas, 720, Meireles, Fortaleza, Ceará. CEP: 60170-020. Telefone: +55 85 98658-0801/85 3037-0801. E-mail: geysammsc@hotmail.com

Conflito de interesses: Não há qualquer conflito de interesses por parte de qualquer um dos autores.

Recebido em: 30 Mai 2017; Revisado em: 22 Ago 2017; Aceito em: 30 Ago 2017. 


\section{INTRODUÇÃO}

Atribui-se a Durston, em 1670, o primeiro registro da atresia de esôfago (AE); embora se credite a Gibson, cerca de três décadas após, a clássica descrição da anomalia. ${ }^{1}$ Datam de 1936, na Inglaterra, por Simpson-Smith, os empenhos iniciais em tratá-la cirurgicamente; e, de 1939, por Leven e Ladd independentemente, a documentação dos primeiros casos bem-sucedidos de reparo. ${ }^{2-3}$

Definida anatomicamente pela interrupção congênita do esôfago e, na maioria dos casos (90\%), acompanhada de fístula traqueal, ${ }^{4}$ reconhece-se hoje a $\mathrm{AE}$ como uma malformação congênita relativamente comum, de incidência 1:2500 a 1:4500 nascimentos, a mais frequente a acometer o órgão. A incidência no sexo masculino revela-se discretamente superior. $^{5}$

Sua etiologia permanece desconhecida, porém, provavelmente multifatorial, dentre os quais se apontam a prematuridade, a gemelaridade, bem como a consanguinidade parental, conforme documentado por Brown em estudo conduzido com 1700 famílias com diagnóstico de pelo menos um dos componentes da associação VACTERL (anomalias da coluna vertebral, atresia anal, anomalias cardiovasculares, fístula traqueoesofágica, atresia esofágica, anomalias renais e defeitos nos membros). ${ }^{6-7}$ Outras linhas de pesquisa investigam ainda o papel da anormalidade de neuropeptídeos no processo de formação do esôfago. Do ponto de vista da contribuição ambiental, questiona-se o papel do consumo materno de dipirona, hormônios sexuais exógenos, tabagismo e alcoolismo materno, diabetes e infecções. ${ }^{8}$

A classificação da AE ocorre mediante localização do sítio de atresia e identificação de fístula traqueal associada, segundo Vogt (1929), modificada por Gross (1953). Apontam-se cinco variantes, cuja compreensão determina o manejo clínico-cirúrgico ${ }^{9-10}$ (Figura 1$){ }^{4}$

Figura 1. Atresia de esôfago: variantes clínicas. (A): Atresia de esôfago sem fístula (8\%); (B): Atresia de esôfago com fístula proximal $(<1 \%)$; $(\mathrm{C})$ : Atresia de esôfago com fístula distal $(87 \%)$; (D): Atresia de esôfago com fístula distal e proximal $(<1 \%)$; $(\mathrm{E})$ : Fístula traqueoesofágica isolada, sem atresia de esôfago (4\%).

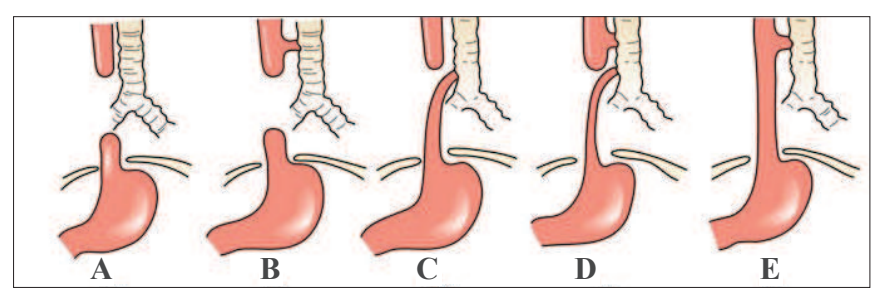

Adaptada de: Townsend CM, Beaucham D, Evers BM, Mattox KL. ${ }^{4}$

A sobrevida dos neonatos com diagnóstico de atresia do esôfago aproxima-se de 95\% em alguns centros, culpabilizando-se a concomitância com outras anomalias congênitas graves, juntamente com o baixo peso ao nascer, pela maior parte dos óbitos. Avanços no suporte nutricional, na assistência ventilatória perioperatória e no manejo clínico intensivo, além da otimização da abordagem anestésico-cirúrgica, constituem alguns dos fatores responsáveis pela otimização das taxas de sobrevida. ${ }^{11}$

Descreve-se a seguir o protocolo da Maternidade Escola Assis Chateaubriand (MEAC), no tangente à propedêutica diagnóstica e terapêutica diante de casos de atresia de esôfago atendidos pela Unidade.

\section{PROTOCOLO}

Os fetos ou neonatos com suspeita clínica de atresia de esôfago atendidos pela MEAC se inserem em uma linha de atenção multidisciplinar - radiologia, obstetrícia, genética, neonatologia, cirurgia pediátrica, anestesiologia, gastroenterologia, enfermagem, nutrição, fonoaudiologia, fisioterapia e terapia ocupacional - que define a estratégia a ser adotada, contemplando desde a investigação diagnóstica até o manejo pós-operatório daqueles candidatos ao reparo cirúrgico.

\section{Diagnóstico pré-natal e conduta obstétrica}

Ainda durante a gestação, possibilitam a suspeição de AE determinados achados ultrassonográficos: ausência ou diminuição de bolha gástrica, escassez de líquido no intestino fetal, dilatação de coto esofágico proximal e, sobretudo, polidramnia com ou sem restrição de crescimento, que se sobressai dentre os demais por ser o mais característico, sobretudo quando aferido posteriormente a vinte semanas de idade gestacional. ${ }^{12-13}$

Gestantes sem sintomas intensos secundários à polidramnia geralmente recebem conduta expectante até a maturidade fetal. Aquelas que os apresentam são internadas para repouso no leito.

A via de parto geralmente obedece às indicações obstétricas, embora malformações fetais potencialmente graves ou complicações da polidramnia (apresentações anômalas, prolapso de cordão ou sofrimento fetal) incrementem os riscos da via vaginal, o que justifica a eleição mais frequente da cesárea. ${ }^{14}$

\section{Diagnóstico neonatal}

Imediatamente após o nascimento, corrobora com a hipótese de AE um conjunto de indicativos clínicos característicos, como a presença de sialorreia aerada persistente em boca e/ ou narina; sinais de comprometimento respiratório (tosse, dispneia/sufocamento, cianose), por vezes acompanhados, à ausculta pulmonar, de roncos disseminados ou estertores subcrepitantes; alteração do aspecto abdominal (distensão ou escavamento) e dificuldade na sondagem orogástrica aspecto bastante típico. ${ }^{15-16} \mathrm{Em}$ certos casos, então, quando não disponíveis exames de imagem no período pré-natal, apenas 
se conjetura a AE mediante dificuldades na amamentação, ao se observarem regurgitação e desconforto respiratório, quando associados aos sinais e sintomas acima descritos (Quadro 1).

Ressalta-se a relevância da radiografia de tórax e abdome após sondagem nasogástrica, que pode revelar parada de progressão em fundo cego da coluna de ar esofágica, indícios de pneumonia, além de anomalias congênitas (vertebrais, cardíacas, dentre outras) não detectadas anteriormente. O recurso do contraste otimiza a identificação do coto esofágico proximal (Quadros 2 e 3 ) (Figuras 2 e 3$).{ }^{17}$

Quadro 1. Protocolo de orientações para sondagem orogástrica em casos suspeitos de AE.

\begin{tabular}{|c|c|}
\hline - & Selecionar sonda orogástrica (SOG) $\mathrm{n}^{\circ} 8$ \\
& Sondagem sem sucesso (Gross $\mathrm{A}-\mathrm{B}-\mathrm{C}-\mathrm{D})$ \\
$\diamond \quad$ Dificuldade de progressão além de $10-12 \mathrm{~cm}$ \\
$\diamond \quad$ Nunca forçar a entrada da SOG \\
- Sondagem com sucesso Gross E (4\% da casuística)
\end{tabular}

Quadro 2. Protocolo de orientações para avaliação radiológica sem contraste.

\begin{tabular}{|c|}
\hline Radiografia panorâmica (visualizar tórax e abdome) \\
$\diamond \quad$ Parada de progressão da sonda orogástrica \\
$\diamond \quad$ Coluna de ar em fundo cego \\
$\diamond \quad$ Indícios de pneumonia \\
$\diamond \quad$ Ausência de ar no trato gastrointestinal \\
$\diamond \quad \begin{array}{l}\text { Anomalias congênitas associadas } \quad \text { (vertebrais, } \\
\text { cardíacas, etc.) }\end{array}$ \\
\hline
\end{tabular}

Quadro 3. Protocolo de orientações para avaliação radiológica com contraste.

\begin{tabular}{|ll|}
\hline 1. & Conectar seringa de 3 ou $5 \mathrm{ml}$ com contraste iodado a SOG no \\
& 8 (preencher todo o trajeto previamente à introdução) \\
2. & Posicionar SOG em coto esofágico proximal \\
3. Sem desconexão da seringa, injetar $0,5 \mathrm{~mL}$ de contraste \\
iodado \\
4. Indícios de pneumonia \\
5. Solicitar radiografia panorâmica (neonato em inclinação $>$ ou \\
$=$ a $\left.45^{\circ}\right)$ \\
6. Logo após a radiografia, aspirar e retirar a SOG sob aspiração \\
contínua
\end{tabular}

Figura 2. Radiografias panorâmicas sem contraste de neonatos com atresia de esôfago: (A) Em detalhe, coluna de ar em fundo cego (setas pretas) e presença de ar em trato gastrointestinal (seta branca). (B) Malformações vertebrais. (C) Ausência de ar no trato gastrointestinal. (D) Pneumonia em pulmão direito.
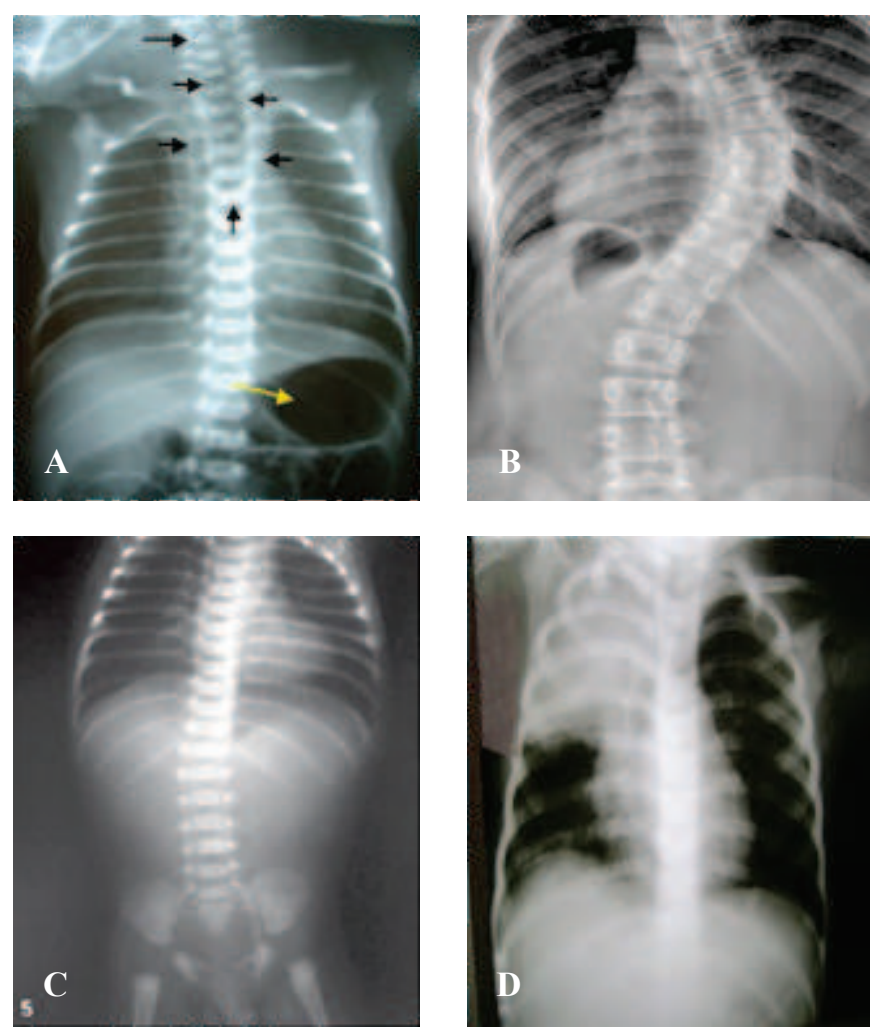

Figura 3. Radiografias panorâmicas com contraste de neonatos com Atresia de Esôfago. Em evidência, o coto esofágico proximal preenchido por contraste iodado radiopaco.
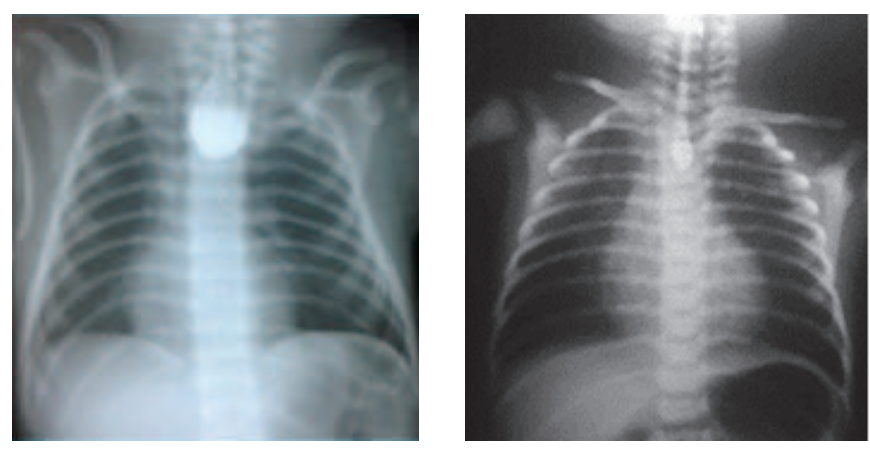

\section{Rotina de cuidados neonatais pré-operatórios}

Uma vez firmado diagnóstico de AE, o rastreio de outras malformações impacta significativamente tanto em tratamento quanto em prognóstico. A precocidade com a qual se inicia o manejo adequado também influencia sobremaneira o prognóstico.

Os distúrbios congênitos associados mais comuns, e também de maior preponderância clínica, são os que afetam o aparelho cardiovascular $(23 \%)$ - defeito do septo interatrial, defeito do septo interventricular, tetralogia de Fallot, persistência do canal arterial, coarctação aórtica ou destro-rotação do seu 
arco. Displasia ou agenesia renais podem ocorrer e requerem destaque. $^{18}$

Para melhor avaliação das malformações anatômicas e consequentemente do planejamento cirúrgico, solicitam-se ultrassonografia abdominal total e ecocardiografia transtorácica (Quadro 4).

A pesquisa de cromossomopatias impõe-se nos pacientes com AE, porquanto expressiva parcela da incidência possui relação com trissomias, sobretudo do 13, 18 e 21, respectivamente, viabilizando o diagnóstico das síndromes de Patau, Edwards e Down. ${ }^{8}$ Assim, acompanhamento adequado com geneticista revela-se salutar.

Após o nascimento, deve-se instalar uma sonda multiperfurada - REPLOGUE - no coto proximal do esôfago, mantida sob aspiração, de modo a evitar a broncoaspiração de saliva (Quadro 5).

Quadro 4. Protocolo de orientações para rotina de avaliação complementar pré-operatória.
- Hemograma, coagulograma
- Ureia, creatinina, sódio, potássio, cálcio séricos
- $\quad$ Glicemia de jejum
- Gasometria arterial
- Radiografia panorâmica (tórax e abdome)
- Ultrassonografia abdominal
- Eletrocardiograma (ECG), Ecocardiografia transtorácica

Quadro 5. Protocolo de orientações para sondagem orogástrica REPLOGUE.

- Mantida sob aspiração continuamente
- Introdução de cateter duplo lúmen 7Fr em SOG nº 10 curta
Conexão de um lúmen em aspiração a vácuo, sob pressão
$60 \mathrm{cmH} 2 \mathrm{O}$
$\quad \begin{aligned} & \mathrm{Se} \text { resíduo espesso, conexão de outro lúmen a infusão } \\ & \text { de soro fisiológico em vazão de } 15-20 \mathrm{~mL} / \mathrm{h}\end{aligned}$

Mantém-se o neonato sob cuidados intensivos, em incubadora aquecida (temperatura entre $36,5-37,4^{\circ} \mathrm{C}$ ), com hidratação e oferta nutricional parenteral, visando a contornar possíveis distúrbios orgânicos (eletrolítico, glicêmico, ácido-base, imunológico, dentre outros) previamente ao procedimento cirúrgico. Para tal, a linha venosa de preferência corresponde ao acesso central de inserção periférica (PICC). A malformação anatômica inviabiliza a introdução de alimentação via oral. Complicações respiratórias - pneumonia, atelectasia (geralmente de lobo superior direito) ou mesmo a síndrome do desconforto respiratório do recém-nascido (30-40\% nasce prematuramente) merecem igualmente atenção nesse período. ${ }^{19}$
Deve-se garantir a permeabilidade de vias aéreas e adequada ventilação, bem como evitar ventilar sob pressão positiva, devido ao risco de distensão gástrica. Procede-se com intubação orotraqueal apenas se houver desconforto respiratório significativo. Opta-se pela posição semi-recumbente no leito (decúbito dorsal com elevação parcial de tronco, aproximadamente $30^{\circ}-45^{\circ}$ ).

O risco inerente ao reparo cirúrgico daAE pode ser estratificado, segundo critérios de peso corporal, status pulmonar e associação de outras anomalias congênitas. A partir disso, determina-se a conduta a ser tomada em cada caso, inclusive no que diz respeito ao agendamento da intervenção. Seguem as classificações de Waterson e Spitz (Quadros 6 e 7). ${ }^{20-22}$

Quadro 6. Classificação prognóstica de Waterson.

\begin{tabular}{|c|l|c|}
\hline Grupo & \multicolumn{1}{|c|}{ Especificação } & $\begin{array}{c}\text { Expectativa } \\
\text { de vida }\end{array}$ \\
\hline A & $\begin{array}{l}\text { Peso acima de 2500g } \\
\text { Ausência de complicações pulmonares } \\
\text { Ausência de malformações associadas }\end{array}$ & \\
\hline B & $\begin{array}{l}\text { Peso entre 1900 e 2500g } \\
\text { Complicação pulmonar discreta } \\
\text { Malformação associada de gravidade } \\
\text { moderada }\end{array}$ & $50-65 \%$ \\
\hline & $\begin{array}{l}\text { Peso abaixo de 1800g } \\
\text { Complicação pulmonar grave } \\
\text { Malformação congênita grave }\end{array}$ & $10-20 \%$ \\
\hline
\end{tabular}

Adaptada de: Waterson DJ, Bonhan-Carter RE, Abedeen E. ${ }^{20}$

Quadro 7. Classificação prognóstica de Spitz.

\begin{tabular}{|c|c|c|c|}
\hline Grupo & Especificação & $\begin{array}{c}\text { Expectativa de } \\
\text { vida em 1994 }\end{array}$ & $\begin{array}{c}\text { Expectativa de } \\
\text { vida em 2006 }\end{array}$ \\
\hline 1 & PN $>1500$ g sem ACM & $97 \%$ & $98 \%$ \\
\hline 2 & PN $<1500$ g ou ACM & $59 \%$ & $82 \%$ \\
\hline 3 & PN $<1500$ g e ACM & $22 \%$ & $50 \%$ \\
\hline
\end{tabular}

Adaptada de: Spitz L, Kiely EM, Morecroft JA, Drake DP. ${ }^{21}$; Lopez PJ, Keys C, Pierro A, Drake DP, Kiely EM, Curry JL, et al. ${ }^{22}$

$\mathrm{PN}$ : peso ao nascimento, ACM: anomalias cardíacas maiores.

\section{Tratamento cirúrgico e manejo anestésico}

Alcançadas otimizações de estado geral e condições clínicas pulmonares, habilita-se o neonato candidato ao reparo cirúrgico, modalidade definitiva de tratamento da AE. Idealmente este pode ser realizado precocemente, a partir de 24-72h após o nascimento, mediante realização do ecocardiograma. Adiamento pode provocar aumento do risco de complicações respiratórias. 
O reparo do esôfago se realiza em intervenção única ou em duas etapas, conforme extensão da atresia e condições clínicas do paciente. Em casos de anatomia favorável, realiza-se ligadura e secção da fístula, usualmente com fios inabsorvíveis, seguidos de anastomose primária do esôfago no qual se utiliza fios absorvíveis. Defeitos mais complexos (long gap) terão conduta individualizada conforme peso e condições clínicas.

A cobertura antibiótica reduz o risco de infecção perioperatória. Se o neonato provém da UTI neonatal e não apresenta sinais de infecção, ou risco por outra condição, administrar profilaticamente, na indução anestésica, cefotaxima ou ampicilina com sulbactam que será mantido por $24 \mathrm{~h}$ após o procedimento. ${ }^{23}$ Se proveniente da UTI neonatal, porém com sinais de infecção, ou risco por outra condição - como rotura de bolsa prolongada - indicado tratamento com ampicilina e gentamicina. Caso o neonato provenha de outro hospital e apresente sinais de infecção, inicia-se antibioticoterapia conforme protocolo da instituição de origem.

$\mathrm{O}$ manejo anestésico direciona-se à ventilação pulmonar dissociada de fuga de fluxo aéreo para a fístula traqueoesofágica. Técnicas empregadas podem compreender, com o paciente em posição semi-recumbente, a intubação acordado ou a indução inalatória com sevoflurano, ambas idealmente sem abolição do esforço ventilatório. ${ }^{24}$ Devem-se ainda evitar bloqueadores neuromusculares e excesso de pressão positiva na ventilação até oclusão cirúrgica da fístula; posteriormente, curarizantes podem então ser empregados com cautela. O correto posicionamento do tubo orotraqueal constitui etapa crítica. ${ }^{25}$

A monitorização utilizada pode englobar cardioscopia, oximetria de pulso, capnografia/capnometria, termômetro nasofaríngeo e pressão arterial invasiva. Opta-se por anestésicos inalatórios na manutenção da hipnose. Repõem-se perdas hídricas insensíveis ou para terceiro espaço com cristaloides aquecidos com fluxo de $6-8 \mathrm{~mL} / \mathrm{kg} / \mathrm{h}$. Coloides (albumina 5\%) também podem ser empregados nos casos de sangramentos de maior vulto.

O profissional responsável deve preparar-se para possíveis intercorrências intraoperatórias, sobretudo de natureza ventilatória, como hipoxemia por seletivação brônquica, obstrução do tubo endotraqueal por secreções, drenagem purulenta, sangramento, acotovelamento traqueal e atelectasia. Redução do retorno venoso e colapso cardiocirculatório também podem ocorrer.

\section{Rotina pós-operatória}

Os neonatos retornam ao centro de terapia intensiva sob sedoanalgesia residual, em ventilação mecânica e flexão cervical para reduzir a tensão sobre a anastomose. Recomenda-se mínimo manuseio do paciente e sedoanalgesia contínua por três a cinco dias com midazolam e fentanil, exceto em prematuros, nos quais o benzodiazepínico pode ocasionar diminuição do fluxo sanguíneo para artéria cerebral média, efeitos neurológicos transitórios (hipertonia, hipotonia, movimentos coreicos, movimentos discinéticos, mioclonia e atividade epileptiforme), além de leucomalácia, hemorragia periventricular e morte. ${ }^{26}$ Preconiza-se ainda o uso de agente curarizante vecurônio durante $72 \mathrm{~h}$; este na dose de ataque de $0,1 \mathrm{mg} / \mathrm{kg}$ e manutenção $0,1 \mathrm{mg} / \mathrm{kg} / \mathrm{h}$, independentemente da tensão na anastomose.

Na presença de sonda através da anastomose, certifica-se do seu correto posicionamento enteral e programa-se início da dieta $48 \mathrm{~h}$ após a cirurgia. Se em posição gástrica, mantê-la aberta, realizar esofagograma entre sete e dez dias de pós-operatório: na ausência de fístula, iniciar dieta via oral; do contrário, manter por mais sete a dez dias a dieta por sonda ou dieta zero e nutrição parenteral e repetir exame, após sete dias, progredindo para dieta enteral quando do seu desaparecimento (Quadro 8).

Quadro 8. Protocolo de orientações para rotina de cuidados pós-operatórios.

- Sedoanalgesia com fentanil e midazolam por três a cinco dias

- Curarização contínua concomitante à sedoanalgesia

$\diamond \quad$ Vecurônio (ataque: $0,1 \mathrm{mg} / \mathrm{g}$ e manutenção $0,1 \mathrm{mg} /$ $\mathrm{kg} / \mathrm{h})$

- Posicionamento semi-recumbente com flexão cervical

- Sonda orogástrica transanastomótica: dieta enteral com 48h de pós-operatório (se bem posicionada). Se posição gástrica: manter aberta, dieta zero e nutrição parenteral (NPT).

- Esofagograma com 7-10 dias

- Dieta oral após sete a dez dias (na ausência de fístulas). Se fistula: 7-10 dias dieta por sonda enteral ou dieta zero e NPT (se posição gástrica) e repete esofagograma com 7-10 dias

- Em caso de perda de sonda não repassar

- Diagnóstico de complicações

$\diamond \quad$ Esofágicas: deiscência, DRGE

$\diamond$ Respiratórias: broncoaspiração, pneumonia, atelectasia

- Imunização: pneumococos e vírus sazonais

- Reabilitação: fonoaudiologia, terapia ocupacional e fisioterapia

O pós-operatório pode cursar com complicações de natureza variada, que envolvem o próprio esôfago - distúrbios na motilidade ou na perviedade do segmento anastomosado. Também potencialmente ocorrem recidiva da fístula traqueal e intercorrências respiratórias, além de escoliose e deformidades da parede torácica. ${ }^{27}$

A dismotilidade esofágica configura achado tardio comum, predispondo à doença do refluxo gastroesofagico (DRGE). Pode advir naturalmente do desenvolvimento neural anormal do esôfago ou da manipulação cirúrgica dos cotos atrésicos, resultando em peristaltismo ineficaz e contrações descoordenadas do segmento distal à reconstrução. ${ }^{28} \mathrm{O}$ reparo cirúrgico e a gastrostomia possivelmente contribuem para o surgimento da DRGE, na medida em que modifica a anatomia da transição gastro-esofágica e do ângulo de His. 
A estenose da anastomose constitui o principal motivo da reabordagem cirúrgica. A maioria dos casos resolve com sessões de dilatação. Fatores de risco mais associados nesse contexto incluem, além da DRGE, a alta tensão ou a deiscência de anastomose dos cotos. ${ }^{29}$

A eficácia do tratamento para DRGE com inibidor de bomba de prótons em recém-nascidos e lactentes abaixo de 11 meses de idade não foi demonstrada, apesar da inibição da secreção de ácido gástrico. ${ }^{30}$ Por esse motivo, essa classe de drogas não faz parte do esquema terapêutico adotado pela MEAC. Geralmente se empregam procinéticos e agentes bloqueadores histaminérgicos tipo II.

Secundariamente à hipertrofia do coto esofágico proximal, pode haver compressão da traqueia e ocorrência de traqueomalácia. Esta dificulta o clearance de secreções, promovendo seu acúmulo em vias aéreas proximais, logo inflamação e proliferação bacteriana local. ${ }^{31}$ Dismotilidade e estenose esofágicas facilitam a broncoaspiração de conteúdo gástrico. A indicação de cobertura antibiótica merece criteriosa avaliação nesse momento. Recomendam-se ainda a otimização das estratégias de imunização, de forma a abranger cepas de pneumococo e vírus sazonais. ${ }^{32}$

Ao longo do processo de recuperação cirúrgica, destaca-se a relevância de exercícios de coordenação sucção-deglutição pela fonoaudiologia e de proteção contra estímulos nocivos e fornecimento de estímulos contingentes pela terapia ocupacional. A fisioterapia participa da reabilitação postural e, sobretudo, respiratória, auxiliando desmame da ventilação mecânica, evitando atelectasias e promovendo toilete das secreções das vias aéreas (Quadro 9).

Quadro 9. Complicações pós-operatórias precoces e tardias.

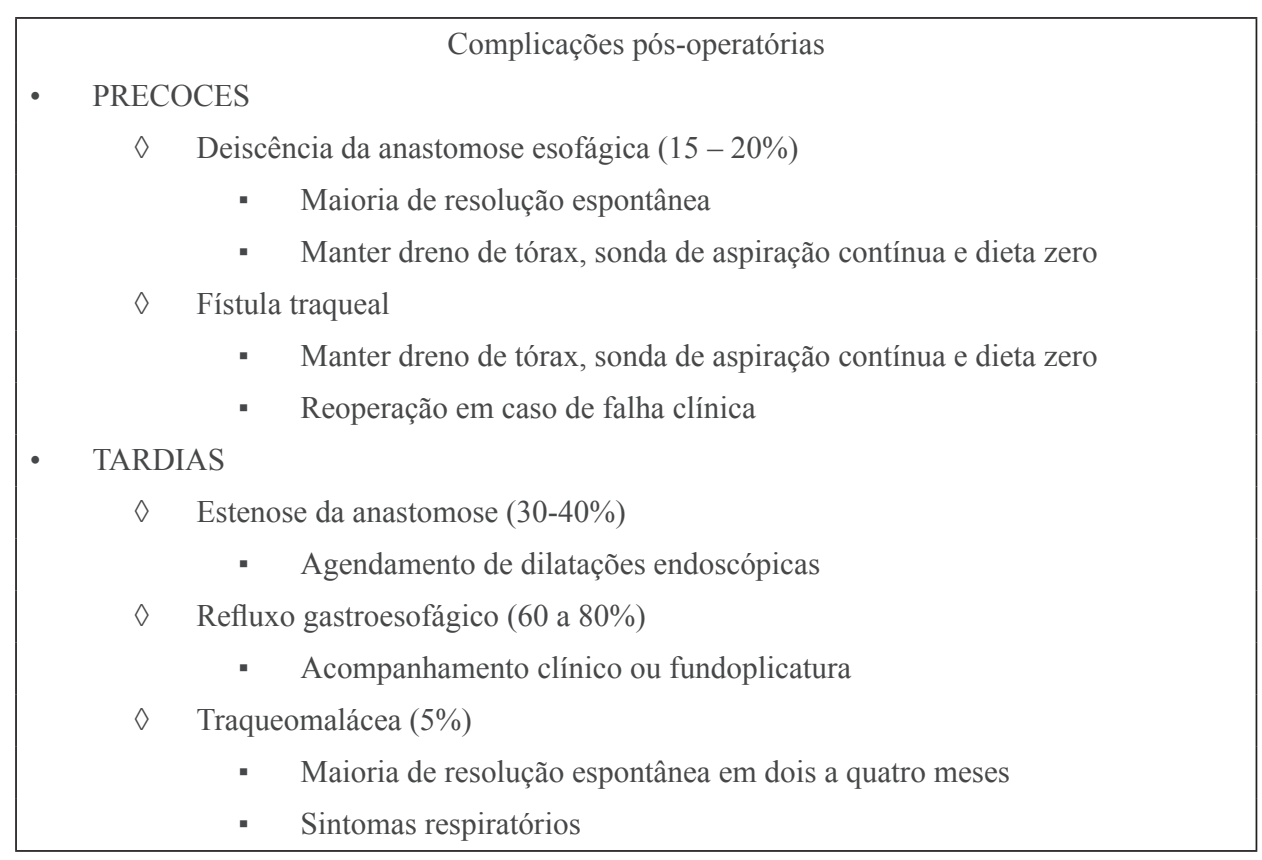

\section{REFERÊNCIAS}

1. Gibson T. The anatomy of humane bodies epitomized. 5 ed. London: Duke University Libraries; 1697.

2. Leven NL, Varco RL, Lannin BG, Tongen LA. The surgical management of congenital atresia of the esophagus and tracheoesophageal. Ann Surg. 1952;136(4);701-17.

3. Ladd WE. The surgical treatment of esophageal atresia and tracheoesophageal fistulas. N Engl J Med. 1944;230:625-37.

4. Townsend CM, Beaucham D, Evers BM, Mattox KL. Sabiston textbook of surgery: the biological basis of modern surgical practice. 18. ed. Philadelphia: WB Sauders; 2007.

5. Nyberg DA; Neilsen IR. Abdomen and gastrointestinal tract. In: Diagnostic imaging of fetal anomalies. Philadelphia: Lippincott Willians \& Wilkins; 2003. p 547-602.
6. Brown AK, Roddam AW, Spitz L, Ward SJ. Oesophageal atresia, related malformations, and medical problems: a family study. Am J Med Genet. 1999;85:31-7.

7. Orford J, Glasson M, Beasley S, Shi E, Myers N, Cass D. Oesophageal atresia in twins. Pediatr Surg Int. 2000;16:541-5.

8. Jong EM. Etiology of esophageal atresia and tracheoesophageal fistula: "Mind the gap". Curr Gastroenterol Rep. 2010;12:215-22.

9. Vogt EC. Congenital esophageal atresia. AJR Am J Roentgenol. 1929;22:463-5.

10. Gross RE. The surgery of infancy and chilhood. Philadelphia: WB Saunders; 1953.

11. Spitz L. Oesophageal atresia. Orphanet J Rare Dis. 2007;2:24. 
12. Holland AJ, Fitzgerald DA. Oesophageal atresia and tracheo-oesophageal fistula: current management strategies and complications. Paediatr Respir Rev. 2010;11:100-6.

13. Houben CH, Curry JI. Current status of prenatal diagnosis, operative management and outcome of esophageal atresia/tracheoesophageal fistula. Prenat Diagn. 2008;28:667-75.

14. Maia NL Filho, Mathias L. Obstetrícia básica. 3. ed. São Paulo: Sarvier; 2006. Capítulo 45, Oligodrâmnio e Poliidrâmnio; p. 352-61.

15. Lahdes-Vasama TT, Sihvonen R, Iber T. Perforation of theupper and lower segments of atretic esophagus (type C) secondary to nasogastric tube insertion. Pediatr Surg Int. 2009;25:537-8.

16. Grosfeld JL, Ladd AP. Anomalias congênitas. In: Silva AC, Pereira RM, Pinheiro PF. Cirurgia pediátrica-condutas clínicas e cirúrgicas. Rio de Janeiro: Guanabara Koogan; 2005. p. 291-8.

17. McDuffie LA, Wakeman D, Warner BW. Diagnosis of esophageal atresia with tracheoesophageal fistula: is there a need for gastrointestinal contrast? J Pediatr. 2010;156:852.

18. Holder TM, Cloud DT, Lewis JE Júnior, Pilling GP. Esophageal atresia and tracheoesophageal fistula. Pediatrics. 1964;34:542-9.

19. Costa CD. Malformações congênitas do esôfago. Rev Fac Ciênc Méd. 2002;4(1-2):38-44.

20. Waterson DJ, Bonhan-Carter RE, Abedeen E. Oesophageal atresia: tracheo-oesophageal fístula. A study of survival in 218 infants. Lancet. 1962;1:819-22.

21. Spitz L, Kiely EM, Morecroft JA, Drake DP. Oesophageal atresia: at risk groups for 1990s. J Pediatr Surg. 1994;29:723-5.

22. Lopez PJ, Keys C, Pierro A, Drake DP, Kiely EM, Curry JL, et al. Oesophageal atresia: improved outcome in high risk groups? J Pediatr Surg. 2006;41(2):331-4.

23. Calil R, Caldas JP. Antibioticoterapia profilatica: quando e como usar. In: Procianoy R, Leone CR, coord. Programa de atualização em neonatologia (PRORN). Porto Alegre: Artmed/Panamericana; 2010. p. $65-88$.

24. Andropoulos DB, Rowe RW, Betts JM. Anaesthetic and surgical airway management during tracheo-oesophageal fistula repair. Pediatric Anesthesia. 1998;8(4):313-9.

25. Maxwell LG. Anesthetic management for newborns undergoing emergency surgery. ASA Refresher Course in Anesthesiology. 2007;35(1):107-26.

26. Harte GJ, Gray PH, Lee TC, Steer PA, Charles BG. Haemodynamic responses and population pharmacokinetics of midazolam following administration to ventilated, preterm neonates. J Paediatr Child Health. 1997;33(4):335-8.

27. Tomaselli V, Volpi ML, Dell'Agnola CA, Bini M, Rossi A, Indriolo A. Long-term evaluation of esophageal function in patients treated at birth for esophageal atresia. Pediatr Surg Int. 2003;19:40-3.

28. Mortell AE, Azizkhan RG. Esophageal atresia repair with thoracotomy: the Cincinnati contemporary experience. Semin Pediatr Surg. 2009;18:12-19.

29. Chittmittrapap S, Spitz L, Kiely EM, Brereton RJ. Anastomotic stricture following repair of esophageal atresia. J Pediatr Surg. 1990;25(5):508-11.

30. Robert MW, Gregory LK. Proton pump inhibitors in pediatrics mechanism of action, pharmacokinetics, pharmacogenetics, and pharmacodynamics. Pediatr Drugs. 2013;15:119-31.

31. Bresci G, Sacco R. Pulmonary or otolaryngologic extraesophageal manifestations in patients with gastroesophageal reflux disease. World J Gastrointest Endosc. 2010;2:47-9.

32. Warris A. Prophylactic antibiotics should be used in children with repaired esophageal atresia/tracheo-esophageal fistula: the case against. Paediatr Respir Rev. 2016;18:62-3.

\section{Como citar:}

Câmera GM, Paes LS, Castro EC, Fernandes TA, Melo AA Filho, Rebelo OB Neto. Atresia de esôfago: diagnóstico perinatal e assistência perioperatória - protocolo da Maternidade Escola Assis Chateaubriand. Rev Med UFC. 2018 jul-set;58(3):84-90. 